\title{
Investigating Data Synchronization in a Mobile Learning Network with Handheld Devices
}

\author{
Vincent Tam \\ Department of Electrical and Electronic Engineering \\ The University of Hong Kong \\ Pokfulam Road, Hong Kong. \\ vtam@eee.hku.hk
}

\begin{abstract}
There were many successful Web-based learning systems built to enhance the effectiveness of institutional teaching over the past decade. Students were benefited in two major aspects: asking questions to their course instructors, and downloading relevant course materials only when Internet/Intranet access is available. Recently, handheld devices integrated with the latest wireless technology are becoming popular. Thus, it is foreseeable that such learning facilities may move beyond the boundary of fixed network connections one day. In this paper, we considered an interesting proposal to mobilize conventional Web-based learning systems with the integration of handheld devices, and pioneered its prototype implementation in the University of Hong Kong. Besides getting encouraging students' feedbacks, we gained invaluable experience of combining frontier technologies such as the Conduits and Web server technology to solve the data synchronization problems of students' questions distributed among the 'local' databases of handheld devices and the centralized database server.
\end{abstract}

Index Terms- handheld devices, web-based learning systems, Palm programming, content synchronization.

\section{INTRODUCTION}

Over the past decade, Web technology has been assimilated into many computer-aided collaborative learning systems such as the Integrated Virtual Leaming Environment (IVLE) [8], the WebCT [9] and the Interactive Leaming Network (ILN) [7] developed by the University of Hong Kong. These systems aim to facilitate instructor-and-students' interactions outside the classroom. The extensive experience in using these learning systems as reported in many research papers $[1,6,9]$ obviously reminded us of their effectiveness as general teaching aids to stimulate outside-classroom interactions. In addition, with the great accessibility of the Internet, these Web-based collaborative learning systems have already been adopted as $e$-classrooms to supplement conventional lectures/tutorials. An illustrative example is the lecture-on-demand (LOD) system with videotaped lectures prepared as video files of different formats downloadable via the Internet to facilitate students' revision. Through these Web-based e-classrooms, students can ask relevant questions to their instructors or copy useful course materials through Internet connections of reasonable speeds

\author{
Barbara Yin \\ Department of Electrical and Electronic Engineering \\ The University of Hong Kong \\ Pokfulam Road, Hong Kong. \\ byin@eee.hku.hk
}

(normally ranging from $56 \mathrm{~K}$ bits-per-second (bps) for dial-up lines to $10 \mathrm{M}$ bps for broadband lines) and affordable costs. However, existing Web-based learning systems still suffer from certain physical limitations such as the fixed number/locations of Internet access points that will adversely affect the accessibility of Web-based learning systems in some cases.

In recent years, the portable communicating and/or computing devices including the Palm ${ }^{\mathrm{m}}$-based Personal Data Assistants (PDAs) [4], the Windows-CE ${ }^{\text {nt }}$-based pocket PCs [3], a wide variety of mobile phones ranging from the ordinary phones, the Wireless Application Protocol (WAP) supported unit to the latest BlueTooth ${ }^{\mathrm{m}}$ devices used as dialup modems for accessing the Internet, and their integration like mobile phones integrated with PDA or Pocket PCs, etc. have been attracting wide popularity and serious attention in the global technology sector. Clearly, the evolution of these portable communicating/computing devices such as mobile phones, pocket PCs and their integration will continuously turn many existing Web applications into truly mobile applications in which portable data devices (such as the Palm" -based PDA) communicating with other handheld devices can selectively invoke the required mobile services such as stock trading or banking transactions provided on secured remote servers (i.e. service mobility) anytime and anywhere. Similarly, the aforementioned Web-based learning facilities such as the students-instructor discussion facility and the course materials downloading function should be developed as a mobile learning system with the availability and popularity of more powerful handheld devices, possibly at a much lower cost in the near future. Imparted with this exciting vision, our research team at the Department of Electrical and Electronic Engineering, the University of Hong Kong (HKU-EEE) initiated a University-funded teaching development project based on the possible uses of handheld devices to facilitate university teaching in late 2001. We named this teaching development project as "the $\underline{H}$ and $\underline{H}$ eld devices for Internal Teaching" (HHIT) [1, 10].

To focus on the two commonly used functions identified on most Web-based learning systems, we proposed the ASKanywhere and STUDY-anywhere subsystems for the targeted mobile learning system of our HHIT project. The 
ASK-anywhere subsystem enables students/instructors to discuss their topics of interest anywhere by synchronizing the original content [4] stored on their handheld devices with any Web-based discussion forum $[6,7,8]$. Any new discussion item entered into a handheld device will be automatically added to the Web-based discussion forum through the content synchronization process. The STUDY-anywhere subsystem allows students the freedom to study their course material anywhere, using relevant course material from the Web-based learning system downloaded onto their handheld devices. Following the recommended practice in Software Engineering, we adopted a systematic phase development approach to build the targeted HHIT system. The $1^{\text {st }}$ phase focused on the basic functionality of the learning system and user interface design. We pioneered a prototype implementation of the HHIT system - Version 1 (HHIT-V1) starting in March 2002, and completed the HHIT-V1 system in September 2002. The detail about HHIT-V2 can be found in $[1,10]$. The $2^{\text {nd }}$ phase emphasized on solving the content synchronization problems of the students' questions stored in the distributed 'local' databases of the handheld devices with the centralized departmental database server through frontier technology such as the Conduits and Web server technology. The prototype of HHIT-Version 2 (HHIT-V2) was just completed in April 2003. The last phase of our HHIT project, mainly to wrap up the project with an automatic installation script, improved features and more detailed documentation, will be completed in the coming June 2003. The complete documentation about our HHIT project should then be available from the authors or the Research Office, the University of Hong Kong on request.

This paper is organized as follows. Section II describes the overall system architecture design of our HHIT system with more detailed discussion about the Conduits and Web server technology used. Section III details the prototype implementation of HHIT-V2 and its empirical evaluation. Lastly, Section IV summarizes our work and sheds light on several directions for future investigation.

\section{HHIT SYSTEM \& SELECTIVE CONTENT SYNCHRONIZATION}

\section{A. The HHIT System Architecture}

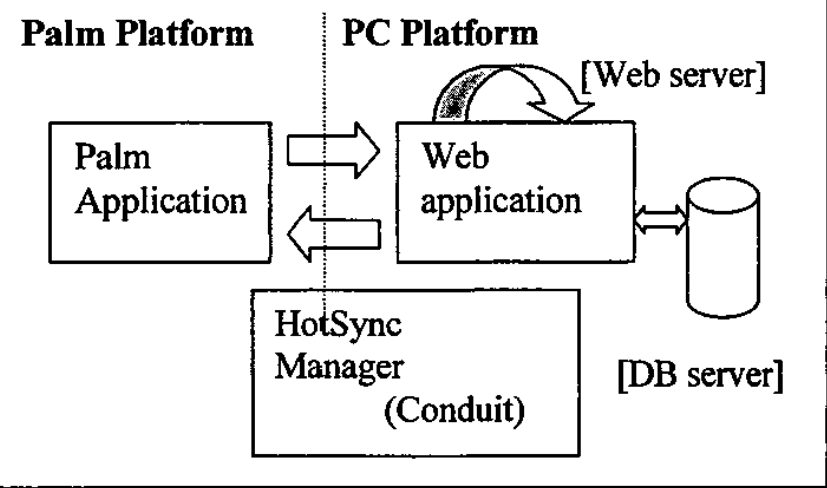

Figure 1. Overall Architecture of the HHIT system.

Figure 1 shows the overall architecture of the HHIT system [1]. It should be noted that all major software components [10], including the Palm application, Web application and application-specific conduit for the HotSync $B$ [4], developed by our team are boxed in Figure 1 while the anxiliary technology such as the Web and Database server are put into square-brackets. Basically, the HHIT system is divided into two main platforms, the Palm and PC platforms, as indicated by the dotted line. The Palm platform involves only one major software component - the Palm application - which divides into the ASK-anywhere and STUDY-anywhere subsystems. The PC platform consists of the Web application, the HotSync Manager ${ }^{(2)}$ [4] with the application-specific conduit, the Web server and the Database server. Similarly, the Web application divided into the ASK-anywhere and STUDY-anywhere subsystems. For each of these subsystems, the corresponding Web application consists of a collection of related Web pages with embedded Java or JavaScript [11] codes to communicate with the Database server to retrieve or update the relevant information - that is "questions" or "course material" stored in the centralized databases.

\section{B. Conduit Programming for Selective Content Synchronization}

A conduit is a customized plug-in module registered with the Palm ${ }^{\text {TM }}$ HotSync Manager application to transfer a userdefined type of data (such as the address records) between the handheld and the desktop computers. Basically, Conduit Programming [4] allows selective content synchronization specified for a user application. Synchronization options including the synchronization direction (i.e. handheld $\Rightarrow \mathrm{PC}$, $\mathrm{PC} \Rightarrow$ handheld or handheld $\Leftrightarrow \mathrm{PC}$ ) and update criteria like the latest file creation time need to be clearly stated in the customized conduit module for a specific Palm application.

As far as HHIT-V2 system is concerned, "selective content synchronization" naturally implies the students should be able 
to choose a particular folder(s), containing either discussion or course materials, to be downloaded into their handheld devices from the Web-based learning systems or vice versa. However, we should be careful that the course materials do not need to be uploaded from students' handheld devices in our HHIT system. Therefore, we provide two different default synchronization options for the ASK-anywhere and STUDYanywhere subsystems. In the ASK-anywhere subsystem, the most updated versions of the FAQs will be transferred between the handheld and Web platforms using the bidirectional (handheld $\Leftrightarrow P C$ ) synchronization mode. On the other hand, the course materials in the STUDY-anywhere subsystem is usually transferred using the uni-directional ( $\mathrm{PC} \Rightarrow$ handheld) content synchronization. Besides, to avoid information loss due to accidental deletion of FAQs on the students' handheld devices, the FAQ synchronization is always done with the "set union" of all the FAQs and their replies collected from both PC and handheld databases. However, in the STUDY-anywhere subsystem, since the centralized PC database always contain the larger and original set of course materials for downloading only, there is no need to perform such "set union" of database records.

Figure 2 shows the basic functionalities of the "customized" conduit of ASK-anywhere subsystem which includes: 1) sending the questions already stored in the Palm PDA to the instructors; 2) receiving the relevant answers form the instructors. In developing our ASK-anywhere subsystem, we chose to use the $\mathrm{C} / \mathrm{C}++$ Sync Suite from the PalmSource [12] since there are relatively more references available on this software tools.

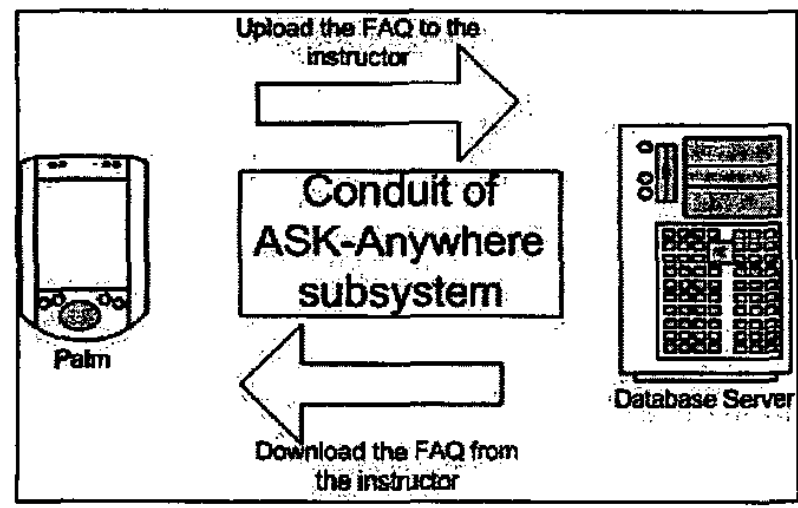

Figure 2. Basic Functions of Conduit for the ASK-anywhere subsystem.

The function of conduit for the STUDY-anywhere is similar to that of the ASK-anywhere subsystem except that there is no conduit for "uploading" for the STUDY-anywhere subsystem. Besides, the Acrobat Reader ${ }^{\text {TM }}$ for Palm ${ }^{\text {TM }}$ has to be preinstalled on handheld devices as the course material viewer in our current HHIT-V2 system.

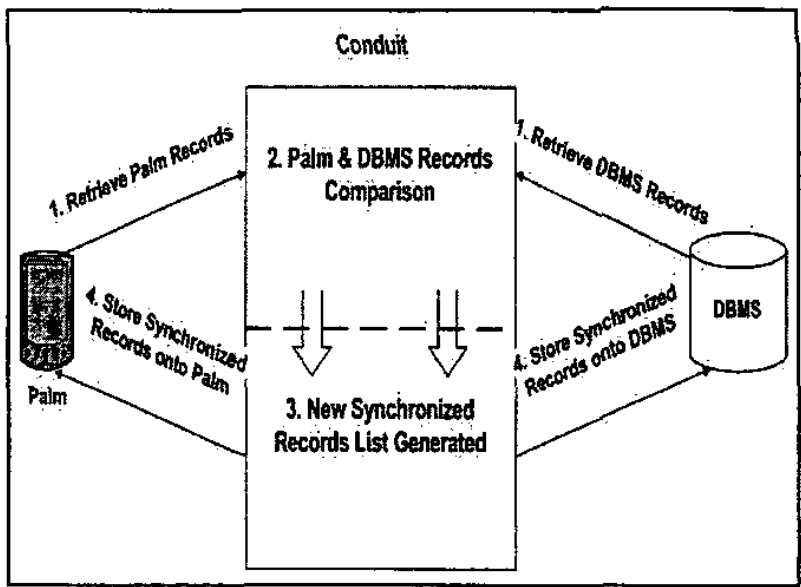

Figure 3. The Detailed Procedure of Content Synchronization for the ASKanywhere subsystem.

Figure 3 details the whole process of content synchronization used in the ASK-anywhere subsystem, which can be described in $\mathbf{4}$ major steps as follows.

1. After user presses on the HotSync icon, the content synchronization between Palm database and DBMS begins;

2. During the 2-way synchronization, records from both Palm database and DMBS are retrieved into the conduit module for comparison first;

3. Record-pairs with the same "FAQ subject title" (or other relevant information) are compared according to the conduit record comparison mechanism which will check whether the relevant records are updated, deleted or added on either side, etc, in a new record list;

4. After the record comparison is completed, a resulting synchronized record list will be generated. The synchronized record list will then be used to update both the Palm (local) database and the DBMS rumning on a centralized database server.

From our experience, the familiarity with the manipulation of the Palm PDA, C/C++ and Palm Programming is essential for Conduit Programming since the Palm PDA has its unique hardware and software architectures with specialized APIs for developing applications. In addition, the reference materials for Palm and Conduit programming are relatively less than those for other programming platforms such as the Web/Windows Programming. For instance, the PalmSource [12] does not provide the sample codes for the interaction between the Palm database, the Palm application and enterprise database server, thus making the task of Conduit programming extremely difficult. After all, we spent 14 manmonths to implement the whole HHIT-V2 system, among which 12 man-months were spent on Conduit Programming. This readily demonstrated the difficulty of Conduit Programming with relatively little documentation available for building our current HHIT-V2 system. 


\section{PROTOTYPE IMPLEMENTATION AND EVALUATION}

We used the CodeWarrior for Palm OS® Version 8.0 [5] as the Integrated Development Environment (IDE) tool to develop all the required Palm applications, such as the "Create New FAQ" and "Reading FAQ" modules in Figure 2. CodeWarrior provides many useful tools, including an editor, project manager, $\mathrm{C} / \mathrm{C}++$ compiler, linker and target device interface that enables source and assembly level debugging to facilitate general Palm application development. In addition, we installed the publicly available Apache ${ }^{\mathrm{TM}}$ server, Jakarta Tomcat $^{\mathrm{TM}}$ engine and Java Development $\mathrm{Kit}^{\mathrm{TM}}$ (JDK) [11] for developing the Web applications. A Palm OS\& emulator [4] was downloaded and used for testing purposes to emulate the hardware of the various models of Palm $\otimes$ powered handheld devices. By running the emulator, we created a "virtual" handheld interface to experiment with the resulting Palm applications of our HHIT prototype system on a desktop computer. The latest prototype HHIT.V2 system is based on the initial HHIT-V1 system developed (September 2002).

We now consider the ASK-anywhere subsystem of the current HHIT-V2 system, followed by a review of the STUDY-anywhere subsystem. Provided is an account of the initial feedback gathered from selected groups of Engineering students in the HKU-EEE department, and a more detailed survey to be conducted early 2003 to evaluate the effectiveness and performance of the HHIT-V2 system. Since launching the project in late 2001, we have received encouraging initial feedback on HHIT-V1 from our Engineering students and researchers sampled.

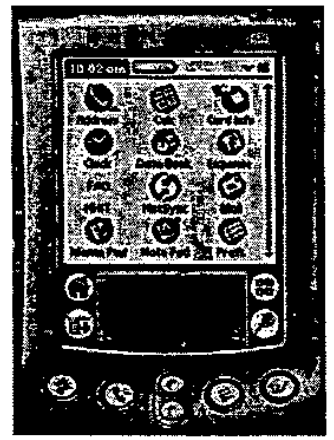

4a. Entry Screen

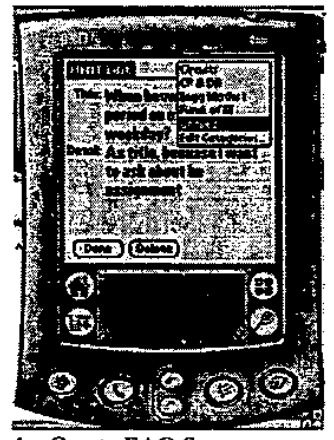

4c. Create FAQ Screen
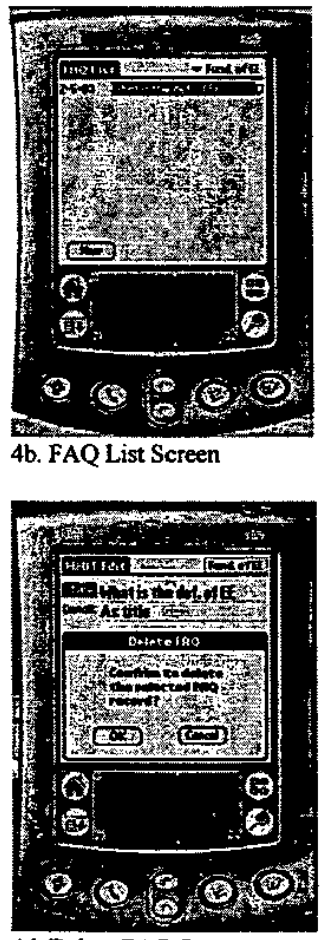

4d. Delete FAQ Screen 4b. FAQ List Screen
Figure 4 shows the user interface of the ASK-anywhere subsystem as viewed on the Palm OS $\$$ emulator:

Figure $4 \mathrm{a}$ is the first entry screen on the Palm PDA where a student clicks the "HHIT" icon to enter the HHIT-V2 system. A list of already downloaded FAQ is shown as in Figure 4b. The student is free to select any of those FAQs to view their detailed content. Furthermore, the student can create a new FAQ on his/her handheld device by clicking the "New" button on the lower left corner of the FAQ List Screen. The student then enters the Create FAQ Screen (Figure $4 c$ ) to create his/her own question to be stored in the Palm FAQ database. Finally, when the instructor's reply to any of the newly created FAQ is downloaded onto the Palm FAQ database, the student selects the reply to view (Fig. 4d).

Lastly, Figure 5a outlines the HHIT-V2 homepage, with a detailed description of its underlying project for the course instructor to login for viewing and answering $F A Q s$, or uploading course materials while Figure $5 \mathrm{~b}$ shows the interface of the InstallShield program to help system administrators in configuring the conduit module properly. For security reasons, our HHIT system is password-protected.

HHIT is an on-going teaching development project; evaluation done to-date is therefore preliminary. We consequently summarize the two major activities for empirical evaluation of the HHIT project as follows:

- Initial evaluation conducted: since our handheld devices project was launched in late 2001, we have randomly selected a sample of 5-10 students from different classes at all levels of Year 1-3, questioning them about their expectation/opinions on handheld devices to facilitate students' learning. Useful suggestions about possible features of our HHIT system have surfaced, (e.g., the FAQ initiator's name be kept anonymous). After our initial HHIT-V1 system [10] was delivered (March 2002), we conducted another series of interviews with students taken from Year $1-2$ classes. At this point we decided to forego detailed surveys so as not to disturb the busy teaching schedule during mid-semester.

- A detailed survey is scheduled to be conducted: after the HHIT-V2 prototype system is officially released for students' trials by the end of April 2002, a more detailed survey is planned to be conducted in June 2003, consisting of an evaluation of many aspects including effectiveness, performance, reliability and security of HHIT-V2.

We will then publish a more complete report on system evaluation. 


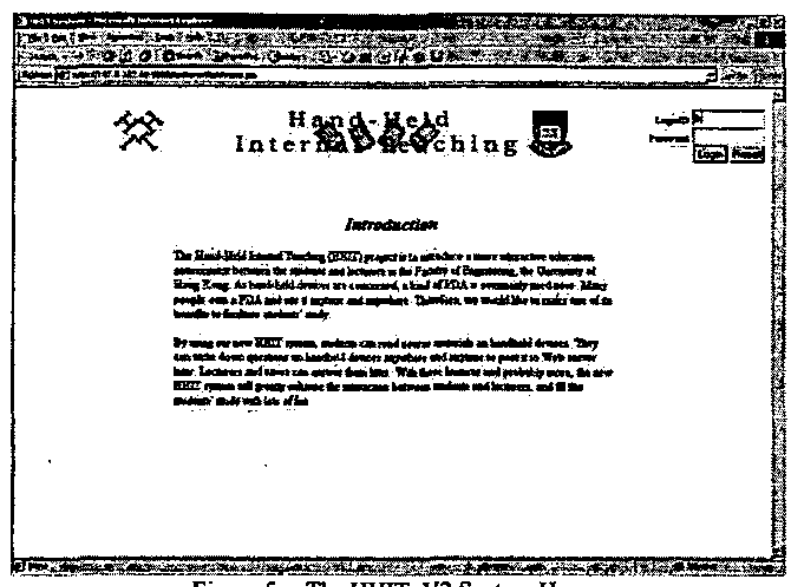

Figure 5a. The HHIT-V2 System Homepage

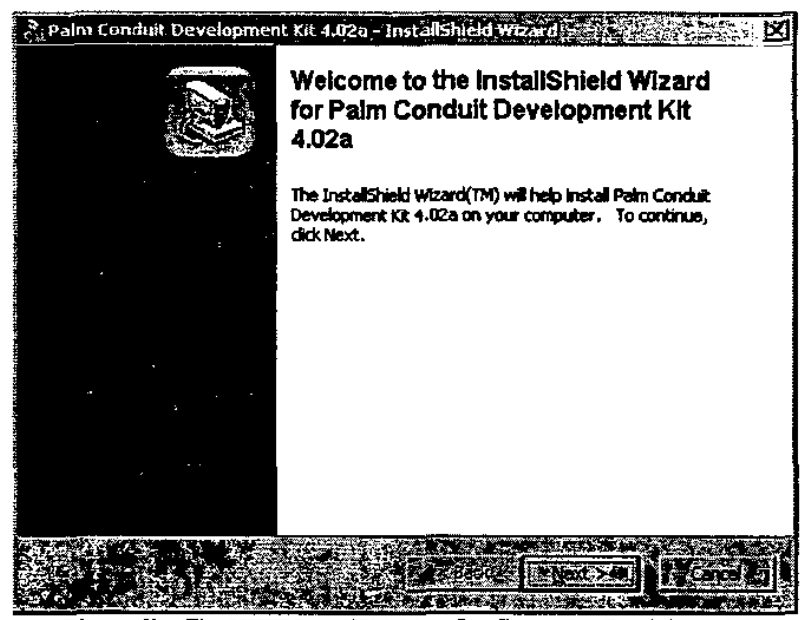

Figure Sb. The HHIT InstallShield to Configure the Conduit module

\section{CONCLUDING REMARKS}

This paper reports an on-going teaching development project - HHIT, conducted in the Department of Electrical and Electronic Engineering at the University of Hong Kong, with increasing progress since November 2001.

Undoubtedly, the HHIT project is important in enhancing the effectiveness of university/college teaching with the frontier Information Technology (IT) or particularly the mobile computing services $[3,4]$ offered by handheld devices. Obviously, with more powerful mobile communication services [2] coming on-line, handheld devices such as the Palm $^{\text {TM }}$ Personal Data Assistant (PDA) $[1,4]$ will definitely become an important user interface to access Web services, and influence our future ways of learning.

There are many possible directions for future investigation. An example is the newly proposed Multicast subsystem of our extended HHIT-II project, which utilizes wireless technology such as Blue Tooth ${ }^{\mathrm{TM}} / \mathrm{W}$ ireless LAN on a handheld device or notebook PC, to act as a communication channel between the instructor and the students. By use of this Multicast system, students post their questions from their handheld devices or notebook computers to their instructor during the lesson or lecture/tutorial. Instructor response can be immediate. The instructor can immediately respond and explain any confusion in greater detail.

\section{Acknowledgment}

The authors acknowledge Mr. James Tsoi and Mr. Ray Tsang for implementing various prototypes of our HHIT system, and the support from the Department of Electrical and Electronic Engineering, HKU. The HHIT project is generously supported by the Internal Teaching Development Fund and the Seed Fund (Applied Research) from the University of Hong Kong.

\section{REFERENCES}

[1] B. Yin and V. Tam. "Extended Learning Access Through Handheld Devices", Proceedings of Ed-Media 2002, Volume 3, pp. 2113 - 2115. ISBN: 1-880094-45-2.

[2] The WAP, 3G, BlueTooth and other mobile technologies, website at http://www.mobilecommercestrategy.com/aa_contents.asp [Last access on November $7^{\text {th }}$ 2002]

[3] CEWindows.NET, website at http:/www.cewindows.net/ [Last access on November $8^{\text {th }} 2002$ ]

[4] Palm.com, website at http://www.palm.com/us/ [Last access on November $8^{\text {th }}$ 2002]

[5] Metrowerks, website at http://www.metrowerks.com/mw/default.htm [Last access on November $8^{\text {th }}$ 2002]

[6] ED-MEDIA, website at http://www.aace.org/conf/edmedia/ [Last access on November $8^{\text {th }} 2002$ ]

[7] Interactive Learning Network (ILN), website at http://iln.cite.hku,hk/LLN/entrance/login.jsp [Last access on November $8^{\text {th }}$ 2002]

[8] Integrated Virtual Learning Environment (IVLE), website at http://ivie.nus.edu.sg, [Last access on November $7^{\text {th }}$ 2002]

[9] WebCT, website at http://www.webct.com [Last access on November $4^{\text {th }} 2002$ ]

[10] The HandHeld devices for Internal Teaching - project or prototype system Version 1.3 (HHIT-V1), website at http://147.8.182.68:8080/HHIT-V1index.html [Last access on November $8^{\text {th }} 20021$

[11] The Java ${ }^{T M}$ Technology, website at http//www.java.sun.com [Last access on November $8^{\text {th }}$ 2002]

[12] The PalmSource, website at http://www.palmsource.com [Last access on December $4^{\text {th }} 2002$ ] 